\title{
AI và nghệ thuật: góc nhìn từ Việt Nam
}

\author{
Hồ Mạnh Toàn
}

SSHPA System

Hà Nội, 20-11-2018

Trong Dead Poets Society (1989), John Keating tuyên bố: "Nhân loại luôn tràn đầy niềm đam mê. Y học, pháp luật, kinh doanh, kĩ thuật là những theo đuổi cao quý cân thiết để duy trì cuộc sống. Nhưng thi ca, sự lãng mạn, tình yêu, vẻ đẹp chính là lí do để chúng ta tồn tại."

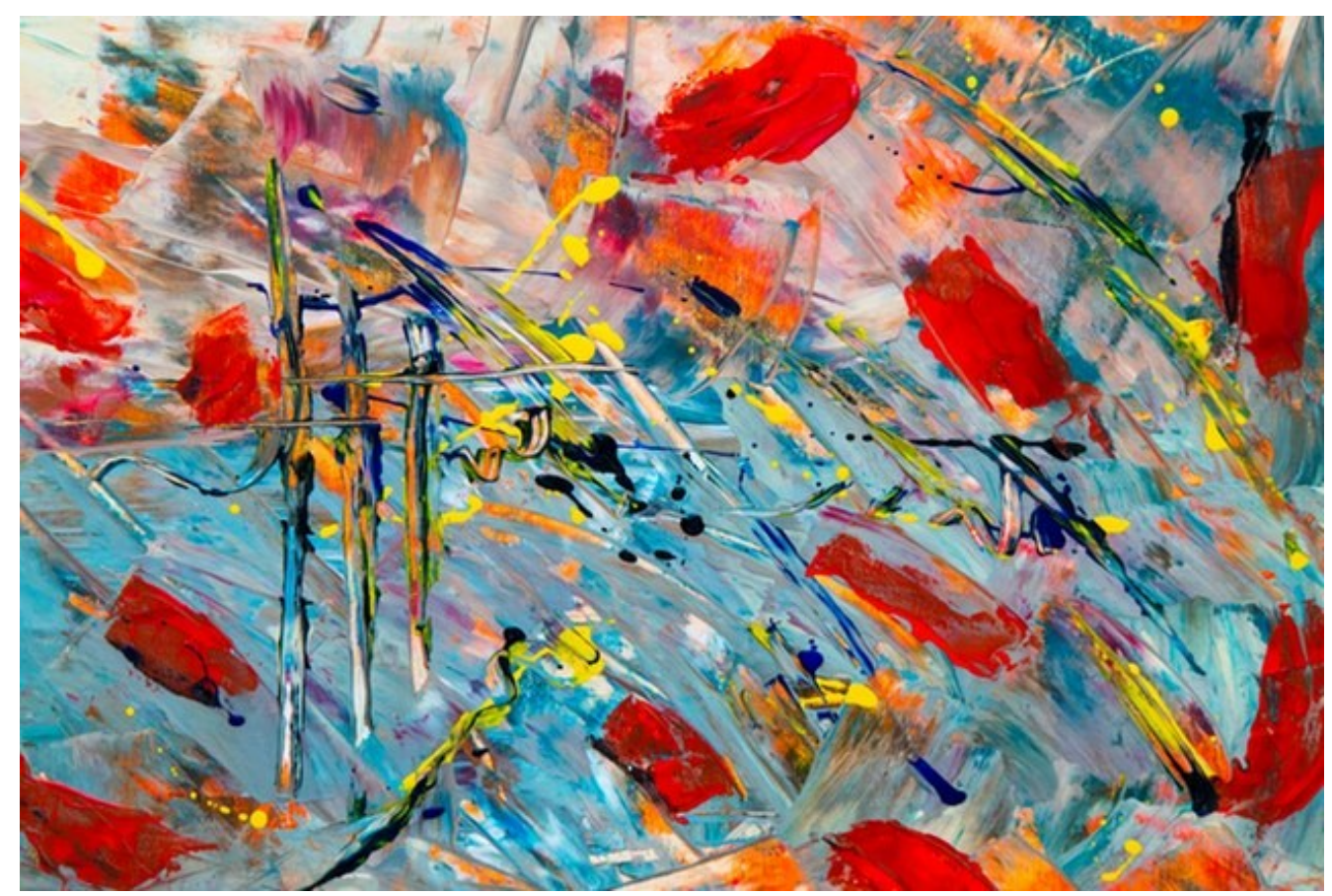

Nguồn: Photo by Steve Johnson from Pexels

Nghệ thuật nói chung và hội họa nói riêng là vẻ đẹp biểu trưng cho sức sáng tạo của con người. Những giá trị văn hóa, lịch sử, nhân văn luôn được thể hiện qua các tác phẩm nghệ thuật. Vì vậy, vẽ tranh bằng thuật toán A.I. đã từng bị coi là thứ "hoang đường" và có phần xâm phạm vào sự cao quý của nghệ thuật. Và bây giờ, một giao dịch thực tế với giá trị lớn đang khiến rất nhiều người phải nghĩ lại.

\section{Thuật toán A.I. vẽ tranh}

Bức tranh Chân dung của Edmond Belamy được bán với giá 432.500 đô la trong một phiên đấu giá cuối tháng 10 tại nhà đấu giá danh tiếng Christie’s. Tại góc phải bên dưới của bức tranh, nơi các họa sĩ thường kí tên, lại kí tên một thuật toán 
máy tính. Chân dung của Edmond Belamy đánh dấu lần đầu tiên một bức tranh với tác giả là một thuật toán được đấu giá và bán thành công [1].

Thị trường tranh từ lâu đã nổi tiếng là một thị trường lớn và ổn định nhưng cũng nhiều điểm mờ vì nhiều động cơ trao đổi mua bán không chỉ đơn thuần là các lý do kinh tế $[2,3]$. Một vài nghiên cứu đã chỉ ra sự thiếu rõ ràng trong cách hoạt động của thị trường nghệ thuật và đã đề xuất một số thay đổi $[4,5,6]$. Tuy vậy, hiện nay hình thức đấu giá vẫn là hình thức phổ biến nhất để trao đổi cũng như đánh giá chất lượng tranh; và hai nhà đấu giá Christie's và Sotheby's vẫn là những địa chỉ uy tín và danh giá.

Chuyện đấu giá và mua bán bức Chân dung của Edmond Belamy đã mang đến nhiều ý kiến trái chiều nhưng không thể phủ nhận, nó là một sự công nhận của một nhà đấu giá uy tín với những phát kiến công nghệ của con người.

\section{Nghệ thuật thời đại số}

Sự phát triển của công nghệ máy tính và sức mạnh của nó trong lĩnh vực xử lí các hình ảnh thực tế không quá xa lạ: từ đơn giản như chọn filter trên Instagram cho đến phức tạp như Photoshop. Hiện nay, mã nguồn mở, lượng data lớn và công nghệ xử lí đồ họa mạnh giúp các kĩ sư máy tính sử dụng A.I. để tạo ra các hình ảnh hay video theo mọi cách có thể mà không cần chụp, quay thực tế. Công cụ Generative adversarial network (GAN) - công cụ đã tạo nên bức tranh được đấu giá tại Christie's - là một ví dụ điển hình với có hai nấc xử lí để đảm bảo chất lượng: generator để học và tạo ra hình ảnh, và discriminator sẽ kiểm tra, đối chiếu với hình ảnh thực tế [7]. Sản phẩm cuối cùng của nó trải dài từ tranh nghệ thuật cho đến mọi phiên bản thời tiết có thể của một bức ảnh phong cảnh.

Khi nói về máy móc, hình ảnh và nghệ thuật, trò chơi điện tử có lẽ nên được xếp vào hàng tiên phong trong việc dung hòa các yếu tố tốt nhất. Sức mạnh xử lí đồ họa của máy tính biến những dòng code trở thành những hình ảnh sống động, một bộ phim tương tác mà người chơi được 'sống' trong nhân vật. ở một mức độ khác, các bộ phim hoạt hình 3D như Toy Story (1995) hay công nghệ bắt chuyển động trong bộ ba Planet of the Apes (2011 - 2017) đều đã phô diễn sức mạnh của công nghệ trong việc khơi gợi cảm xúc của người xem.

Điều khác biệt giữa công nghệ đồ họa máy tính và công nghệ A.I. là sự phân định rõ rệt giữa công cụ và tác giả, giữa 'máy móc là nghệ sĩ' hay 'máy móc là công cụ hỗ trợ' [8]. Phần thông tin tác phẩm nhằm tôn vinh đội ngũ làm ra một bộ phim hay một trò chơi chưa bao giờ khẳng định đóng góp của thuật toán máy tính rõ rệt như cách mà bức tranh Chân dung Edmond Belamy đã làm. Máy móc về bản chất không có tính xã hội hay nhân văn như chúng ta vẫn hiểu để có thể thực sự 'tạo ra' một tác phẩm nghệ thuật [9]. 


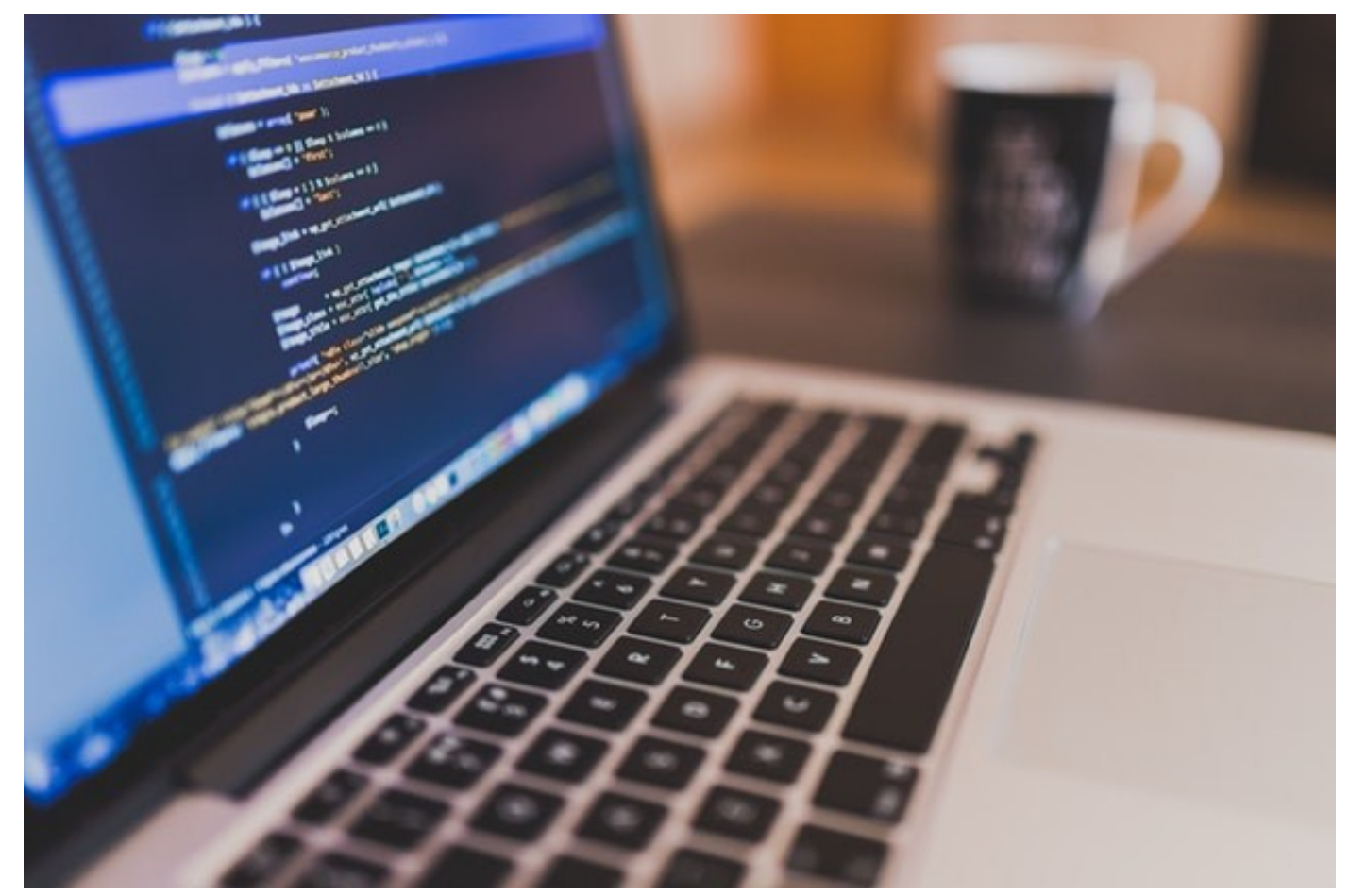

Nguồn: $\underline{\text { Pexels }}$

Câu chuyện văn hóa đằng sau mỗi bức tranh chính là điều tạo nên giá trị của chúng. Trái lại, các bức tranh do A.I. tạo ra thường gây choáng ngợp bởi câu chuyện kĩ thuật mà thiếu một câu chuyện đậm tính nhân văn để níu giữ cảm xúc của người xem.

Có những sự hoài nghi nhất định về $\mathrm{AI}$ và nghệ thuật nhưng các chuyên gia đều coi công nghệ này sẽ là một công cụ quan trọng đối với các nghệ sĩ và công việc sáng tạo nghệ thuật trong tương lai. Sự thay đổi lớn sẽ nằm ở mối quan hệ giữa máy móc và con người: người nghệ sĩ không tự tay thực hiện công việc sáng tạo mà thông qua thuật toán để sáng tạo [10], hay khi máy móc có khả năng đem đến những đóng góp bất ngờ như một người đồng nghiệp [8]. Đồng thời, trí thông minh nhân tạo cũng vượt xa khỏi ranh giới của một công cụ vì nó là một dạng trí thông minh [11], và sử dụng trí thông minh nhân tạo cũng tương tự việc dạy máy móc suy nghĩ một cách nhân văn [12].

\section{Góc nhìn từ Việt Nam}

Nền mỹ thuật Việt Nam đã chứng minh các tác phẩm nghệ thuật luôn gắn liền với các giá trị văn hóa, truyền thống của Tam Giáo của mình $[13,14,15]$. Ví dụ những bức tranh khắc gỗ đầu tiên có mục đích tâm linh rõ rệt. Giai đoạn đương đại và hiện đại của mỹ thuật Việt Nam sau đó có sự tiếp thu các kĩ thuật, tư tưởng nghệ thuật của người Pháp. Tuy nhiên các họa sĩ Việt Nam đều cố gắng truyền tải những chủ đề Việt Nam nhất qua tác phẩm của mình như làng quê, thiên nhiên, phố cổ Hà Nội. 
Tính truyền thống Á Đông cao kết hợp với kĩ thuật vẽ hiện đại cho đến nay vẫn là đặc điểm quan trọng của hội họa Việt Nam. Dù đã có một số họa sĩ hiện đại thử nghiệm với các thể loại mới nhưng thành công vẫn rất khiêm tốn. Đồng thời nền mỹ thuật Việt Nam vẫn còn nhiều vấn đề tồn đọng như tranh giả, cơ chế hay triết lý [13]. Chính vì thế, dù tranh Việt Nam vẫn rất đẹp và giá tranh lên cao nhưng chưa có nhiều sự phá cách mới mẻ thực sự gây tiếng vang lớn.

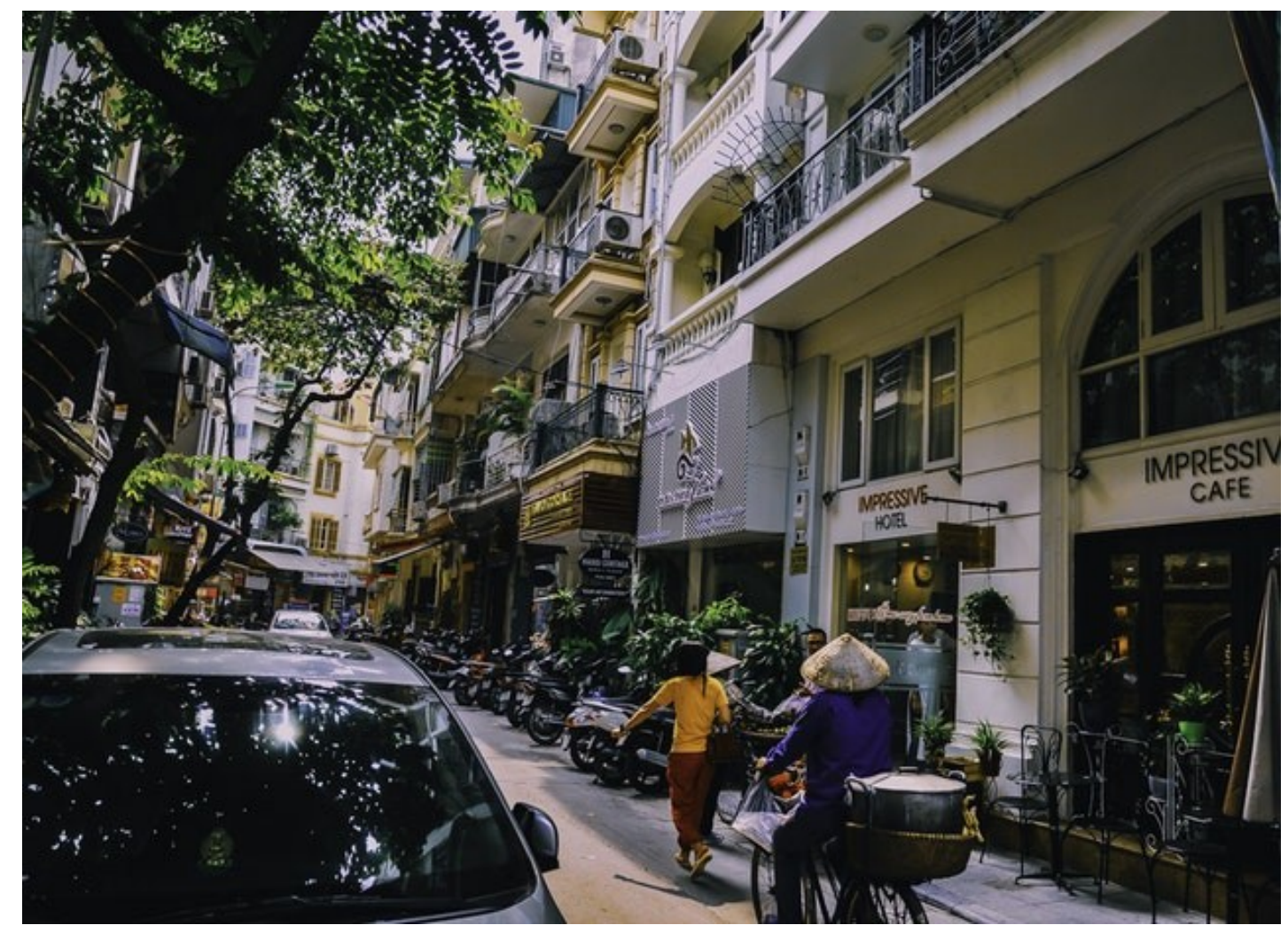

Nguồn: Photo by Arnie Chou from Pexels

Chính vì thế, áp dụng một công cụ mới như A.I vào hội họa sẽ cần thêm nhiều thời gian. Để có thể tận dụng hết sức mạnh của A.I. cần kiến thức về thuật toán, máy tính và lập trình. Trong khi đó, khi nhắc đến vẽ tranh thì hầu hết các công cụ truyền thống vẫn quen thuộc hơn với các họa sĩ. Câu chuyện này cũng tương tự như sự khởi đầu của trí tuệ nhân tạo trong ý khoa khi những ý tưởng đầu tiên xuất phát từ phòng lab máy tính, và bản thân các bác sĩ cũng mất nhiều thời gian để thực sự chấp nhận nó [16]. Dù ứng dụng công nghệ, cách mạng 4.0 đang được nhiệt liệt ủng hộ tại Việt Nam nhưng với tính đặc thù cao của hội họa, Việt Nam cần có sự chuẩn bị về mặt kĩ thuật, cũng như lối suy nghĩ liên ngành để có thể thực sự tham gia vào cuộc chơi A.I. trong nghệ thuật, hội họa.

Tuy vậy, trước mắt công nghệ A.I. có thể là một cứu cánh hỗ trợ các cơ quan chức năng giải quyết các vấn đề về quản lí hay tranh giả. Hiện nay, việc làm giả tranh 
tại Việt Nam khá là phức tạp và chưa có các hướng giải quyết hợp lí, đặc biệt là trong thời đại mạng xã hội tràn lan. Chính vì thế, có thể một cơ sở dữ liệu danh cho họa sĩ và các tác phẩm của họ sẽ giúp cho việc quản lí dễ dàng hơn. Ngoài ra, các ứng dụng A.I. trong phát hiện tranh giả đã được một số quốc gia, bảo tàng nước ngoài áp dụng và hỗ trợ rất nhiều cho các chuyên gia.

Công nghệ hiện nay xuất hiện sâu trong đời sống của người dân Việt Nam thông qua điện thoại thông minh hay mạng xã hội. Tuy nhiên, chúng ta lại chưa có sự chuẩn bị chu đáo với các ảnh hưởng dài hạn và sâu sắc mà công nghệ A.I. có thể mang đến cho nghệ thuật, hội họa.

\section{References:}

[1] Christie. (2018). Is artificial intelligence set to become art's next medium?. Retrieved from URL: https://www.christies.com/features/A-collaborationbetween-two-artists-one-human-one-a-machine-9332-1.aspx.

[2] Horvitz, J. E. (2009) An overview of the art market. In Collectible Investments for the High Net Worth Investor. Edited by Stephen Satchell. Oxford: Elsevier, pp. 85-117.

[3] Plattner, S. (1996). High art down home: An economic ethnography of a local art market. Chicago and London: University of Chicago Press.

[4] David, G., Oosterlinck, K., \& Szafarz, A. (2013). Art market inefficiency. Economics Letters, 121(1), 23-25.

[5] Kräussl, R., Lehnert, T., \& Martelin, N. (2016). Is there a bubble in the art market?. Journal of Empirical Finance, 35, 99-109.

[6] Day, G. (2014). Explaining the Art Market's Thefts, Frauds, and Forgeries (and Why the Art Market Does Not Seem to Care). Vanderbilt Journal of Entertainment and Technology Law 16, 457-95.

[7] Rothman, J. (2018). In the age of A.I., is seeing still believing?. The New Yorker. Retrieved from URL: https://www.newyorker.com/magazine/2018/11/12/ in-the-age-of-ai-is-seeing-still-believing.

[8] Lomas, A. (2018). On hybrid creativity. Arts, 7(3), 25, doi:10.3390/arts7030025.

[9] Hertzmann, A. (2018). Can computers create art?. Arts, 7(2), 18, doi:10.3390/arts7020018

[10] Edmonds, E. (2018). Algorithmic art machines. Arts, 7(1), 3, doi:10.3390/arts7010003.

[11] Arcars, B. A. Y. (2017). Art in the age of machine intelligence. Arts, 6(4), 18, doi:10.3390/arts6040018.

[12] Smith, G. W., \& Leymarie, F. F. (2017). The machine as artist: An introduction. Arts, 6(2), 5, doi:10.3390/arts6020005.

[13] Vuong, Q.H., Ho, M.T., Vuong, T.T., Tran, K., \& Ho, M. (2018b). "Paintings can be forged, but not feeling": Vietnamese art-Market, fraud, and value. Arts, 7(4), 62, doi:10.3390/arts7040062. 
[14] Vuong Q. H., Tran T. D. (2009). The cultural dimensions of the Vietnamese private entrepreneurship. The IUP Journal of Entrepreneurship Development, 6(3/4): 54-78.

[15] Vuong Q. H., La V. P., Vuong T. T., Nguyen V. H., Ho M. T., Nguyen T. H. K., Bui Q. K., Ho M. T. (2018). Cultural additivity: Behavioural insights from the interaction of Confucianism, Buddhism, and Taoism in folktales. Palgrave Communications, 4, DOI: 10.1057/s41599-018-0189-2. URL: https://www.nature.com/articles/s41599-018-0189-2.

[16] Shortliffe, E. H. (1993). The adolescence of AI in medicine: Will the field come of age in the '90s?. Artificial Intelligence in Medicine, 5, 93-106. 